\title{
Tumor-induced mortality in adult primary supratentorial glioblastoma multiforme with different age subgroups
}

\author{
Chang Shu ${ }^{1,2}$, Xiaoling Yan ${ }^{3}$, Xuebin Zhang ${ }^{3}$, Qiong Wang ${ }^{1}$, Sen Cao ${ }^{4}$ \& Jinhuan Wang *, 1,2 \\ ${ }^{1}$ Tianjin Cerebral Vascular \& Neural Degenerative Disease Key Laboratory, Tianjin Neurosurgery Institute, Department of \\ Neurosurgery, Tianjin Huan Hu Hospital, Tianjin 300350, PR China \\ ${ }^{2}$ School of Medicine, Nankai University, 94 Weijin Road, Tianjin 300071, PR China \\ ${ }^{3}$ Department of Pathology, Tianjin Huan Hu Hospital, Tianjin 300350, PR China \\ ${ }^{4}$ Tianjin Zhongtianchi Software Technology Development Co., Ltd, Tianjin 300210, PR China \\ *Author for correspondence: huanhuwangjinhuan@126.com
}

\begin{abstract}
Aim: To assess the independent determinants of tumor-induced mortality in different age subgroups after considering competing risk (CR). Methods: Data were extracted from the SEER database. The independent determinants of tumor-induced mortality were defined by CR analysis and validated by conditional inference trees. A CR nomogram was created based on the proportional subdistribution hazard model. Results: The different age subgroups had their own independent determinants of tumor-induced mortality. Using these variables, a CR nomogram was built with good discrimination and calibration. Conclusion: When conducting population-based cohort studies, a CR analysis is recommended for cancers with short survival and high mortality. A CR nomogram represents the first attempt at a predictive model for quantifying tumor-induced mortality.
\end{abstract}

First draft submitted: 17 September 2018; Accepted for publication: 10 January 2019; Published online: 18 March 2019

Keywords: competing risk $\bullet$ machine learning $\bullet$ nomogram $\bullet$ primary glioblastoma $\bullet$ survival

Primary glioblastoma multiforme (GBM) has a median age at diagnosis of 62 years and accounts for approximately $90 \%$ of all GBM cases [1]. GBM remains almost invariably fatal, with short survival and high mortality despite current optimal surgical and chemoradiotherapy regimens [2]. GBM is most commonly located in the supratentorial region, with the highest incidence in a single lobe, followed by multiple lobes [3]. For many years, patient age has been known to be strongly related to prognosis [4]. However, the impacts of demographic and clinical-related factors on patient mortality across age groups from a population-based dataset have not been completely established.

According to a population-based cohort study, a considerable number of patients may die from competing risk (CR) causes, such as natural causes or cancer-unrelated diseases, especially elderly patients. In the analysis of CR data, traditional survival analysis methods (e.g., Kaplan-Meier and Cox regression analyses) always lead to incorrect and biased results [5]. Thus, we provide a series of CR analysis methods to estimate cumulative incidence, compare cumulative incidence curves and assess the prognostic significance of a given factor after adjusting for other risk factors. Additionally, CR nomograms were built for different age subgroups. At present, no such predictive tool that is applicable to individual GBM patients is available. Therefore, the predictive nomograms will facilitate the counseling of patients from different age subgroups in terms of tumor-induced mortality and subsequent clinical follow-up.

\section{Methods}

Patient enrollment \& characteristics

We performed this population-based retrospective cohort analysis using data from the SEER database, which was designed and is maintained by the National Cancer Institute (USA). To obtain a homogeneous cohort of adult patients with primary supratentorial GBM (PS-GBM), a total of 38,049 patients diagnosed with GBM between January 2000 and December 2013 were identified (SEER ICD-0-3 code: 9440/3, 9441/3, 9442/3). The inclusion

Future Medicine 
Table 1. Demographic and clinical-related characteristics of the different age subgroups.

\begin{tabular}{|c|c|c|c|c|c|}
\hline Characteristic & N.Age1 (\%) & N.Age2 (\%) & N.Age3 (\%) & $x^{2}$ & $p$-value \\
\hline Sex: & & & & 206.4 & $<0.001$ \\
\hline - Male & $5377(62.74)$ & $5583(56.99)$ & $1010(46.27)$ & 3343.8 & $<0.001$ \\
\hline - Female & $3193(37.26)$ & $4214(43.01)$ & 1173 (53.73) & 1674.9 & $<0.001$ \\
\hline Chemotherapy: & & & & 25.115 & $<0.001$ \\
\hline - Pre-TMZ & $2429(28.34)$ & $2617(26.71)$ & $598(27.39)$ & 1322.5 & $<0.001$ \\
\hline - TMZ & $2586(30.18)$ & $2766(28.23)$ & $613(28.08)$ & 1435.1 & $<0.001$ \\
\hline Surgery: & & & & 1152.5 & $<0.001$ \\
\hline - No & $1130(13.19)$ & $1967(20.08)$ & 990 (45.35) & 409.76 & $<0.001$ \\
\hline - STR & $4216(49.19)$ & 4650 (47.46) & 759 (34.77) & 2834.2 & $<0.001$ \\
\hline - GTR & $3224(37.62)$ & $3180(32.46)$ & $434(19.88)$ & 2241.4 & $<0.001$ \\
\hline Radiotherapy: & & & & 1475.4 & $<0.001$ \\
\hline$-\geq 5$ & 3765 (43.93) & $4076(41.60)$ & $916(41.96)$ & 2078.2 & $<0.001$ \\
\hline Vital status: & & & & 482.19 & $<0.001$ \\
\hline - Alive & $1550(18.09)$ & $1022(10.43)$ & 77 (3.53) & 1261.4 & $<0.001$ \\
\hline - Die of GBM & $6719(78.40)$ & 8228 (83.98) & 1937 (88.73) & 3833.3 & $<0.001$ \\
\hline - Die of others & $301(3.51)$ & $547(5.58)$ & $169(7.74)$ & 217.13 & $<0.001$ \\
\hline SES (tertile): & & & & 7.5524 & 0.1094 \\
\hline - Tertile 1 & $2030(23.69)$ & $2356(24.05)$ & $490(22.45)$ & 1222.3 & $<0.001$ \\
\hline - Tertile 2 & $2828(33.00)$ & 3350 (34.19) & $764(35.00)$ & 1616.2 & $<0.001$ \\
\hline - Tertile 3 & $3712(43.31)$ & 4091 (41.76) & $929(42.56)$ & 2048.4 & $<0.001$ \\
\hline SES (quintile): & & & & 18.291 & 0.019 \\
\hline -White & 7591 (88.58) & 8962 (91.48) & 2031 (93.03) & 4349.5 & $<0.001$ \\
\hline - Black & $491(5.73)$ & $399(4.07)$ & $58(2.66)$ & 329.36 & $<0.001$ \\
\hline - Others & $488(5.69)$ & $436(4.45)$ & $94(4.31)$ & 270.04 & $<0.001$ \\
\hline Marital status: & & & & 281.43 & $<0.001$ \\
\hline - Unmarried & $5758(67.19)$ & $6832(69.74)$ & $1115(51.08)$ & 4042 & $<0.001$ \\
\hline - Married & $2812(32.81)$ & $2965(30.26)$ & $1068(48.92)$ & 973.49 & $<0.001$ \\
\hline Tumor site: & & & & 2.1425 & 0.3426 \\
\hline - Unilobal & $6716(78.37)$ & $7750(79.11)$ & $1703(78.01)$ & 3881.8 & $<0.001$ \\
\hline - Multilobal & $1854(21.63)$ & $2047(20.89)$ & 480 (21.99) & 999.91 & $<0.001$ \\
\hline
\end{tabular}

and exclusion criteria are shown in Supplemental Figure 1. Patients were divided into three age subgroups by the $\mathrm{x}-$ tile program (Yale University, CT, USA) to obtain the optimal cut-off value for prognosis [6]. The following patient characteristics were analyzed: gender, chemotherapy, resection extent, radiotherapy, tumor size, socioeconomic status (SES) tertiles, SES quintiles, race, marital status and tumor site (a detailed category for each characteristic is shown in Table 1). Chemotherapy was represented by the following three calendar periods according to previous documents [7,8]: January 2000-February 2005 (pre-temozolomide [TMZ] and pre-bevacizumab [BEV], P1), March 
2005-April 2009 (post-TMZ and post-BEV, P2) and May 2009-December 2014 (post-TMZ and post-BEV, P3). SES tertiles and SES quintiles were defined as SES indexes, which are time-dependent composite scores. The SES index can reflect a person's socioeconomic status [9]. The categories of tumor sizes for the different age subgroups were chosen based on the x-tile program and document [10].

\section{Statistical analysis}

Patient variables (containing each subcategorical variable) were compared among the different age subgroups using $\chi^{2}$ tests. Follow-up was defined as the number of months from diagnosis to either death or censoring. Death from PS-GBM and death from other causes were the primary end points of interest in the analysis. The end point date of the follow-up was November 2016. For the CR analysis, we used the cumulative incidence function to generate a cumulative incidence curve and a kappa-sample test to compare the CIFs for a univariate analysis across the category groups ( $\mathrm{R}$ package 'cmprsk'). The $\mathrm{CR}$ regression model was built using the variables obtained by the univariate analysis. A subdistribution hazard ratio (sHR) and a 95\% confidence interval (CI) were obtained to understand the strength of the relative risk of a given factor after adjusting for other risk factors and after considering the CR. To minimize the influence of information loss, a backward stepwise regression was performed after the CR regression analysis ( $\mathrm{R}$ package 'crrstep'). The Akaike information criterion (AIC) was used as the selection criterion to distinguish between the different CR regression models, and a lower AIC value suggested relative superiority. To validate the accuracy of the CR analysis, we used another machine-learning method with a completely different principle design to eliminate confounding factors for the overall survival (OS) analysis, which was obtained using a Kaplan-Meier method and a Cox regression analysis. Use of a conditional inference tree (ctree), which is a recursive binary partitioning machine learning algorithm, can overcome the possible overfitting and selection bias toward covariates produced by a traditional survival analysis. The covariates contributed nothing to the purity of the subgroups and were considered confounding factors for patient survival ( $\mathrm{R}$ package 'partykit'). Any sHR or HR $>0.1$ showed an increased risk of tumor-induced death or overall death. $\mathrm{p}<0.05$ was considered statistically significant.

\section{CR nomogram design}

CR nomograms for predicting the probability of tumor-induced death were built with proportional subdistribution hazard models [11]. One-third of the cases in the total cohort were selected at random as an internal validation cohort. An internal bootstrap cross-validation machine learning method was performed to correct the overfitting bias that resulted from testing on the same population cohort (R package "riskRegression"). The area under the receiver operating characteristic curve (AUC) was a measure of discrimination in this study [12]. The AUCs were compared using the Delong-Delong test. The calibration plot, in which the predicted and observed probabilities fell along a $45^{\circ}$ line, was used to evaluate the calibration of the nomogram. The risk score for each individual patient was calculated by totaling the points of every variable listed in the nomogram.

\section{Results}

\section{Patients \& treatment}

A total of 20,550 PS-GBM patients were extracted from the SEER database. A total of $87.1 \%$ of them died during the observation period, among which 16,884 (94.3\%) died from the primary tumor, and 1017 died from secondary causes (Supplemental Figure 2). The median OS and tumor-specific survival (TS) for PS-GBM patients were both 8 months. The mean follow-up period was $14 \pm 18$ months. The mean onset age of PS-GBM in the patient cohort of 20,550 individuals was 62 years (both male and female). The gender distribution was 11,970 males $(58.2 \%)$ and 8580 females $(41.8 \%)$. The ethnicity was predominantly white $(91 \%)$. The tumor gross total resection and subtotal resection were performed in $6838(33.3 \%)$ and $9625(46.8 \%)$ patients. A total of 13,701 (66.7\%) patients received radiotherapy during the disease process. For chemotherapy, 5644 (27.5\%), $5965(29 \%)$ and 8941 (43.5\%) patients were diagnosed in the P1, P2 and P3 periods, respectively. In this study, the x-tile program identified optimal age cut-off values and divided the cohort into three different age subgroups: 18-59 years (age1), 60-78 years (age2) and $\geq 79$ years (age3) based on the OS and TS analyses. The differences in OS and TS of each age subgroup were statistically significant (Supplemental Figure 3; each p-value $<0.001$ ). The distributions of demographic or clinical-related characteristics, which are listed in Table 1, were significantly different across the three age subgroups, except for the SES tertiles and the tumor site. After we categorized each characteristic into the different subgroups, the distributions of all factors were significantly different across the three age subgroups. The total median OS and 


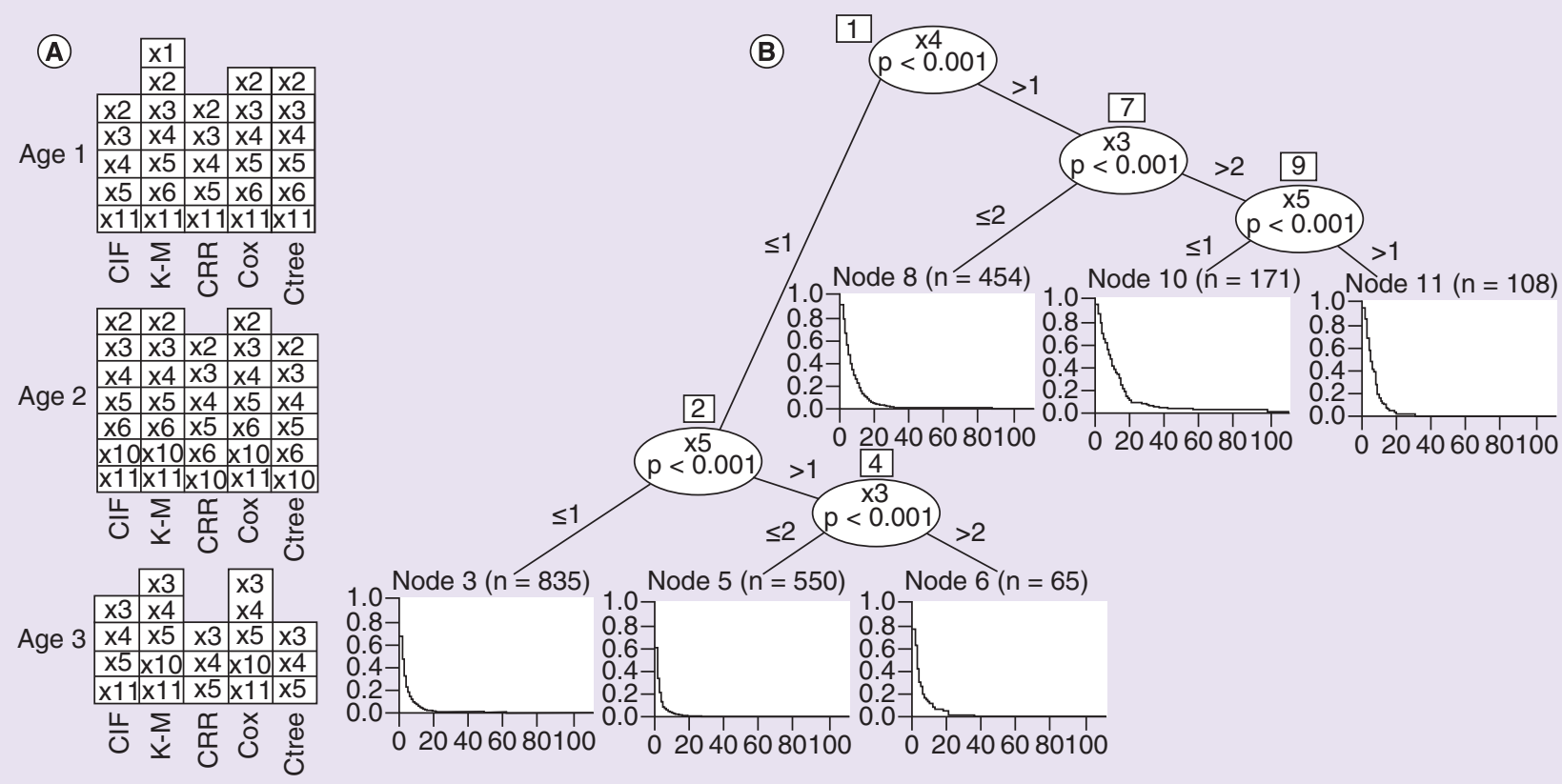

Figure 1. Variables selection. (A) Independent determinants of tumor-induced mortality obtained by different univariate and multivariate analyses. (B) Decision tree for the age3 subgroup was built using a ctree algorithm (the decision trees of age 1 and age 2 subgroups were the same as that of the age3 subgroup).

CIF: Cumulative incidence function; Cox: Cox regression; CRR: Competing risk regression; ctree: Conditional inference trees; K-M: Kaplan-Meier method; x2: Chemotherapy; x3: Resection extent; x4: Radiotherapy; x5: Tumor size; x6: SES tertiles; x10: Marital status; x11: Tumor site.

TS for the age1, age 2 and age 3 subgroups were 15,8 and 3 months, respectively, for the median OS and 16, 8 and 3 months, respectively, for the median TS. The median survivals for each of the categories in the three age subgroups are demonstrated in Supplemental Table 1.

\section{Independent determinants of tumor-induced mortality}

The deaths from CR causes, as a percentage of total deaths for each age subgroup, were age1: 4.29\%, age2: $6.23 \%$ and age $3: 8.02 \%$. Although the incidence of CR causes accounted for less than $10 \%$ in the different age subgroups, the independent determinants obtained by the multivariate CR analysis were less than those obtained by the traditional multivariate survival analysis. In this study, the smallest AICs were achieved when all covariates selected by the multivariate analyses were incorporated. For validation purposes, we used the ctree algorithm to select the independent determinants; the results were the same as those obtained from the CR analysis in the age 2 and age 3 subgroups (Figure 1); in the age1 subgroup, only one independent determinant was not included in the $\mathrm{CR}$ analysis. We concluded that the final independent determinants of tumor-induced mortality obtained by the $\mathrm{CR}$ analysis were more suitable for use in the CR nomograms. Table 2 shows the final independent determinants of tumor-induced mortality selected to use in making the CR nomograms.

\section{CR nomograms}

Plots of the CR nomograms are shown in Figure 2. The AUC values of the age 1, age 2 and age 3 training cohorts were 81.7 (95\% CI: 79.6-83.7), 83.4 (95\% CI: 81.2-86.9) and 79.6 (95\% CI: 75.9-81.2), respectively, in predicting tumor-induced mortality at 6 months, whereas the AUC values of the age1, age 2 and age 3 internal validation cohorts were 81.8 (95\% CI: 89.8-83.8), 83.1 (95\% CI: 80.3-86.0) and 78.6 (95\% CI: 75.9-81.2), respectively. The AUC values of the age 1, age 2 and age 3 training cohorts were 76.5 (95\% CI: 72.9-80.0), 79.3 (95\% CI: 73.0 85.6) and 73.5 (95\% CI: 69.8-77.2), respectively, in predicting tumor-induced mortality at 12 months, whereas the AUC values of the age1, age2 and age 3 internal validation cohorts were 76.6 (95\% CI: 73.0-80.1), 79.2 (95\% CI: 72.9-85.5) and 73.7 (95\% CI: 70.0-77.5), respectively. The AUC values of the age1, age2 and age 3 training 
Table 2. Variables selected for use in making the competing risk nomograms for three different age subgroups after multivariate survival analyses.

\begin{tabular}{|c|c|c|c|c|c|c|}
\hline \multirow[t]{2}{*}{ Selected variance } & \multicolumn{3}{|c|}{ Competing risk regression } & \multicolumn{3}{|c|}{ Cox $\mathrm{PH}$ regression } \\
\hline & p-value & sHR & $95 \% \mathrm{Cl}$ & p-value & HR & $95 \% \mathrm{Cl}$ \\
\hline \multicolumn{7}{|l|}{ Age group1 } \\
\hline \multicolumn{7}{|l|}{ Chemotherapy: } \\
\hline - Pre-TMZ & $<0.001$ & 1.304 & $1.227-1.386$ & $<0.001$ & 1.2703 & $1.1980-1.3470$ \\
\hline$-\mathrm{TMZ}$ & $<0.001$ & 1.125 & $1.062-1.192$ & 0.0012 & 1.0998 & $1.0382-1.1650$ \\
\hline - TMZ-BZM & & 1 & & & 1 & \\
\hline \multicolumn{7}{|l|}{ Surgery: } \\
\hline$-\mathrm{No}$ & $<0.001$ & 1.267 & $1.115-1.440$ & 0.001 & 1.1826 & $1.0701-1.3070$ \\
\hline - STR & $<0.001$ & 1.245 & $1.183-1.310$ & $<0.001$ & 1.2573 & $1.1941-1.3239$ \\
\hline - GTR & & 1 & & & 1 & \\
\hline \multicolumn{7}{|l|}{ Radiotherapy: } \\
\hline - Untreated & $<0.001$ & 1.574 & $1.423-1.741$ & $<0.001$ & 1.7806 & $1.6505-1.9209$ \\
\hline - Treated & & 1 & & & 1 & \\
\hline \multicolumn{7}{|l|}{ Size $(\mathrm{cm})$ : } \\
\hline$-<5$ & 0.028 & 0.946 & $0.900-0.994$ & 0.0335 & 0.9497 & $0.9056-0.9960$ \\
\hline$-\geq 5$ & & 1 & & & 1 & \\
\hline \multicolumn{7}{|l|}{ Tumor Site: } \\
\hline - Unilobal & 0.0041 & 0.913 & $0.857-0.971$ & $<0.001$ & 0.8951 & $0.8449-0.9483$ \\
\hline - Multilobal & & 1 & & & 1 & \\
\hline \multicolumn{7}{|l|}{ Age group2 } \\
\hline \multicolumn{7}{|l|}{ Chemotherapy: } \\
\hline - Pre-TMZ & $<0.001$ & 1.353 & $1.281-1.428$ & $<0.001$ & 1.3502 & $1.2829-1.4210$ \\
\hline$-\mathrm{TMZ}$ & $<0.001$ & 1.156 & $1.096-1.218$ & 0.0016 & 1.0850 & $1.0314-1.1415$ \\
\hline - TMZ-BZM & & 1 & & & 1 & \\
\hline \multicolumn{7}{|l|}{ Surgery: } \\
\hline- No & $<0.001$ & 1.227 & $1.114-1.352$ & $<0.001$ & 1.2257 & $1.1354-1.3231$ \\
\hline - STR & $<0.001$ & 1.209 & $1.154-1.266$ & $<0.001$ & 1.3006 & $1.2390-1.3653$ \\
\hline - GTR & & 1 & & & 1 & \\
\hline \multicolumn{7}{|l|}{ Radiotherapy: } \\
\hline - Untreated & $<0.001$ & 1.736 & $1.611-1.871$ & $<0.001$ & 2.3282 & $2.1935-2.4713$ \\
\hline - Treated & & 1 & & & 1 & \\
\hline \multicolumn{7}{|l|}{ Size $(\mathrm{cm})$ : } \\
\hline$-<5$ & $<0.001$ & 0.883 & $0.843-0.924$ & $<0.001$ & 0.8517 & $0.8162-0.8887$ \\
\hline$-\geq 5$ & & 1 & & & 1 & \\
\hline \multicolumn{7}{|l|}{ SES: } \\
\hline - Tertile 1 & $<0.001$ & 1.180 & $1.113-1.251$ & $<0.001$ & 1.3034 & $1.2351-1.3755$ \\
\hline - Tertile 2 & $<0.001$ & 1.097 & $1.043-1.153$ & $<0.001$ & 1.1516 & $1.0969-1.2030$ \\
\hline - Tertile 3 & & 1 & & & 1 & \\
\hline \multicolumn{7}{|l|}{ Marital: } \\
\hline - Married & 0.031 & 0.945 & $0.898-0.995$ & $<0.001$ & 0.8354 & $0.7981-0.8744$ \\
\hline - Unmarried & & 1 & & & 1 & \\
\hline \multicolumn{7}{|l|}{ Age group3 } \\
\hline \multicolumn{7}{|l|}{ Surgery: } \\
\hline$-\mathrm{No}$ & 0.022 & 1.170 & $1.023-1.339$ & 0.0210 & 1.170 & $1.024-1.34$ \\
\hline - STR & 0.0009 & 1.189 & $1.074-1.317$ & 0.0011 & 1.186 & $1.070-1.31$ \\
\hline - GTR & & 1 & & & 1 & \\
\hline \multicolumn{7}{|l|}{ Radiotherapy: } \\
\hline - Untreated & $<0.001$ & 1.601 & $1.434-1.788$ & $<0.001$ & 1.605 & $1.437-1.79$ \\
\hline - Treated & & 1 & & & 1 & \\
\hline Size $(\mathrm{cm})$ : & & & & & & \\
\hline$-<5$ & 0.014 & 0.897 & $0.822-0.978$ & 0.0019 & 0.871 & $0.798-0.95$ \\
\hline$-\geq 5$ & & 1 & & & 1 & \\
\hline
\end{tabular}




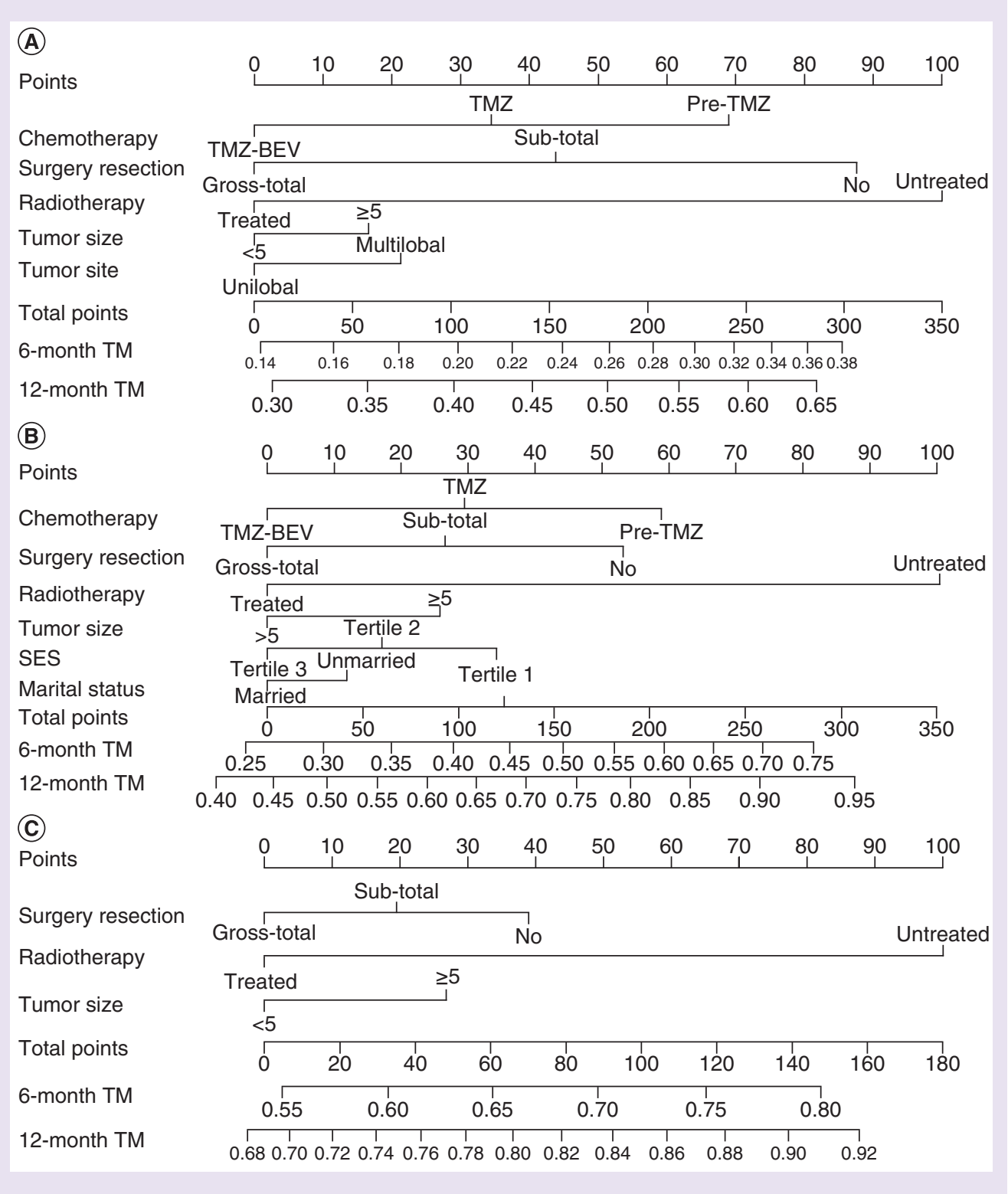

Figure 2. Nomograms. Competing risk nomograms for the prediction of the risk of tumor-induced death in the age1 (A), age2 (B) and age3 (C) subgroups.

BEV: Bevacizumab; SES: Socioeconomic status; TMZ: Temozolomide.

cohorts were 68.6 (95\% CI: 62.2-75.1), 71.5 (95\% CI: 54.6-88.3) and 63.4 (95\% CI: 59.3-69.6), respectively, in predicting tumor-induced mortality at 24 months, whereas the AUC values of the age 1 , age 2 and age 3 internal validation cohorts were 68.5 (95\% CI: 62.0-74.9), 71.4 (95\% CI: 64.6-88.2) and 63.1 (95\% CI: 59.2-69.4), respectively. The AUC values obtained from the internal validation cohorts maintained a level similar to those from each training cohort. This result demonstrated that our nomograms produced good discrimination capability. However, the mean AUC values in predicting tumor-induced mortality at 24 months were less than 70 . Therefore, we only selected 6 and 12 months for plotting the CR nomograms. The calibration plots presented excellent agreement between the nomogram prediction and the actual observation for the 6-and 12-month cumulative incidences (Figure 3). 

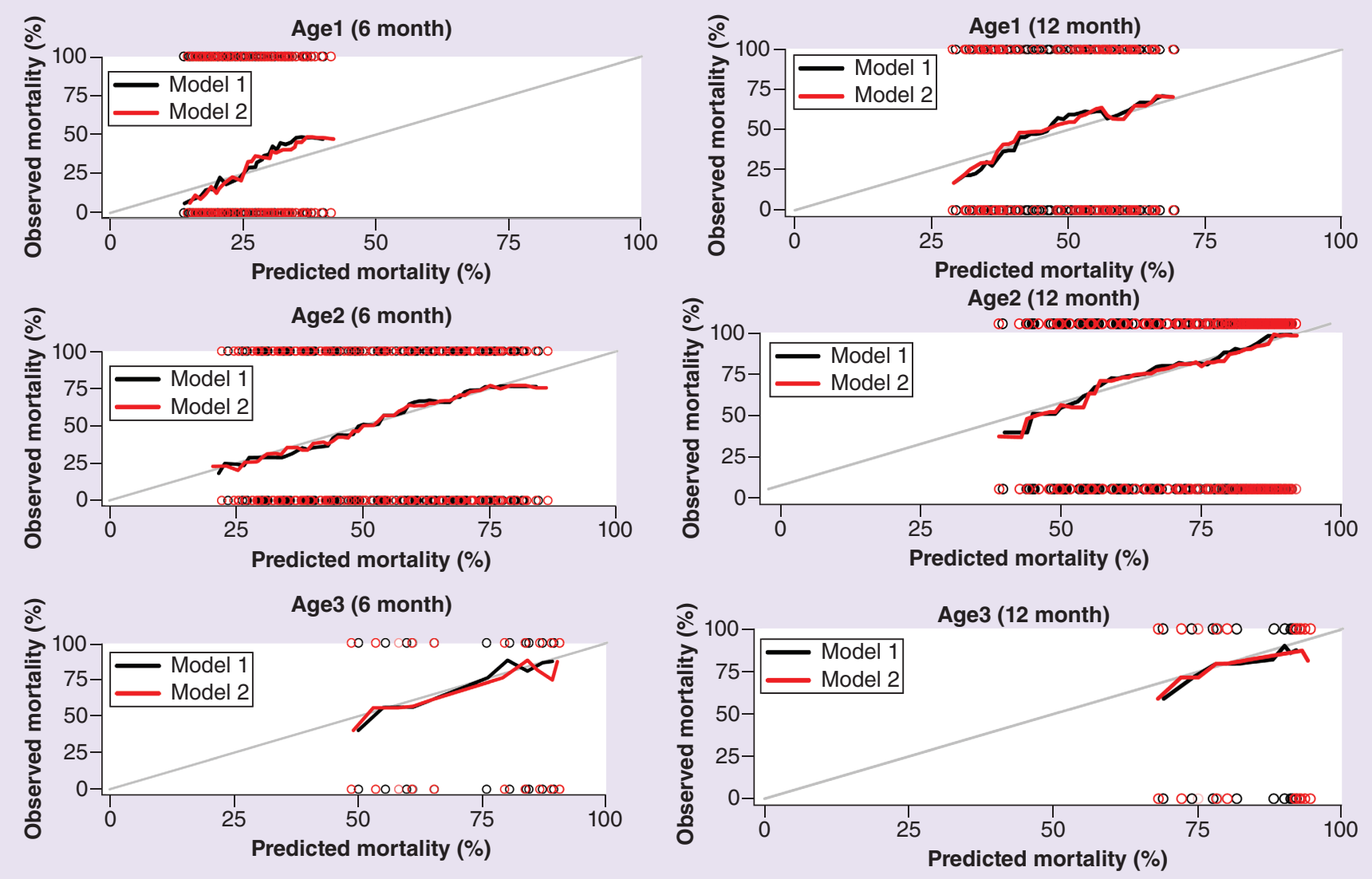

Figure 3. Calibration curve for predicting tumor-induced mortalities. At both 6 and 12 months in the training cohort (red line) and the internal validation cohort (line black) for the age1, age2 and age3 subgroups.

\section{Discussion}

A competing event either blocks the observation of the event of interest or modifies the chance that this event occurs [13]. The CR for the event of interest is not conformed to the independent censoring assumption for a traditional survival analysis [14]. Therefore, to eliminate the incorrect and biased conclusion obtained by a traditional survival analysis, the CR analysis has been applied in mortality studies for head and neck squamous cell carcinoma, colon cancer, thyroid cancer and stage I non-small-cell lung cancer [15-18]. A common feature of these cancers is their optimal 5-year survival rates, which endow these cancers with a high incidence of CR as time passes, such as deaths from heart disease and cerebrovascular disease. However, CR analysis for cancers with short survival and high mortality, such as primary GBM, has not been reported. Because the incidence of CR is low, many studies of primary GBM have replaced tumor-induced death with overall prognosis $[10,19]$. However, the impact of competitive risk factors on the results cannot be determined unless CR analysis is performed (Supplemental Figure 4). In our study, we used a SEER dataset to provide a large population-based cohort, which made it possible to do a CR analysis. After validation by a ctree algorithm, the CR analysis obtained the robust independent determinants of tumor-induced mortality compared with a traditional survival analysis, especially for patients $\geq 60$ years of age (age2 and age3 subgroups).

Older patients are often underrepresented in clinical cancer research. For example, the pivotal clinical trial that established the current standard treatment for patients with newly diagnosed GBM (which used radiotherapy with concurrent TMZ, followed by adjuvant TMZ) excluded patients $>70$ years of age [19]. The SEER dataset provided a large population-based PS-GBM cohort with an age range from 18 to 100 years, which made it possible to explore the survival statuses of elderly patients. We divided the whole cohort into three age subgroups with different independent determinants of tumor-induced mortality, among which the age 2 and age 3 subgroups comprised elderly patients. The age 1 , age 2 and age 3 subgroups represented young (18-59 years), old (60-78 years) and older 
patients ( $\geq 79$ years), respectively, in this paper. Our results showed that aggressive surgery and radiotherapy were related to the decreasing cumulative incidences of tumor-induced death for the different age subgroup patients. As such, elderly patients, even in their eighth or ninth decade of life, can also acquire survival benefits from aggressive surgery and radiotherapy. Our results further supported the conclusions that elderly patients tolerate aggressive surgeries without increased surgery-related morbidity and that they have prolonged survival compared with similar patients undergoing needle biopsies and subtotal resections [20], and radiotherapy prolongs survival compared with supportive care alone, without compromising the quality of life in elderly patients with GBM [21]. The risk of GBM-induced death was significantly reduced during the P2 time period and was further decreased in the P3 time period for both young and old patients but did not differ significantly among older patients. This finding might partly be due to a decreased number of older patients and a lower power for the detection of differences. The most likely explanation is that patients who are in their eighth or ninth decade of life have poorer physiological reserves, more medical comorbidities and a decreased ability to tolerate chemotherapy-induced neurotoxicity.

Our research confirmed that patients with a tumor size $<5 \mathrm{~cm}$ had a lower rate of tumor-induced mortality than patients with a tumor size $\geq 5 \mathrm{~cm}$, regardless of which age subgroups they belonged to. We suggest that surgeons should strive to remove as much of the tumor as possible to decrease the tumor size, and patients should obtain early access to cancer diagnostic services before the tumor size has increased. The prognostic effects of tumor site were only observed in young patients. Young patients possess relatively longer clinical courses than elderly patients, which increases the chances of recurrence. Under this context, a multilobe PS-GBM that is thought to have extremely aggressive characteristics [22] will increase the rate of recurrence and accelerate patient death. Several studies have reported that marital status is an independent prognostic factor for GBM [23,24]. However, none of these studies performed an age-stratified analysis. In our research, we detected that marital status does not predict tumor-induced mortality in young or older patients, but it does predict tumor-induced mortality in old patients. A possible explanation for this finding is that people have become increasingly serious about their marital status when they first enter old age. During this stage, a marriage gives older patients more mental and physical support. The role of SES in GBM survival prediction remains controversial. Researchers who support this conclusion believe that the effects of SES on survival time could extend beyond the effects of access to care and treatment received [25]. However, opponents of this conclusion have declared that SES does not affect prognosis in patients with GBM [26]. After the analysis of the age subgroups was done, we suggested that SES indeed plays an important role in predicting the risk of GBM-induced death as well as overall death, but this effect was only significant in the old patient subgroup, not in the young or older patient subgroups. The reason for this result may be that young patients typically get financial support from their family, which will confound their own SES, and GBM in older patients is rapidly progressive and ultimately fatal, which makes the association between SES and tumor-induced mortality uncertain. This evidence suggests that more family and social support should be given to old patients to decrease tumor-induced mortality.

The CR nomograms were created for the different age subgroup patients. The nomogram provides a quantified prognosis for individual patients and helps in determining treatment decisions for patients and their families [27]. Our CR nomograms relied on easily available parameters from demographic and clinical-related characteristics and achieved an AUC value of more than 80 for the tumor-induced mortality prediction at 6 months. Therefore, the CR nomograms are valuable prognostic tools for GBM patients from different age subgroups.

Study limitations mainly arose from the observation nature of the SEER data. These limitations are described in detail in many published papers $[23,25]$. Therefore, conclusions cannot be drawn about causality or specific clinical care decisions. In addition, we used a bootstrap approach to evaluate the CR nomogram performance. Although this approach was effective, external validation with other cohorts is still needed to estimate model accuracy. However, using strict inclusion and exclusion criteria, we established a homogeneous cohort of PS-GBM patients to perform an age subgroup analyses. We believe that our findings shed new light on detecting independent determinants of tumor-induced mortality in GBM patients. More importantly, the CR analysis is recommended to analyze short survival and high mortality cancers using population-based cohorts to decrease the incidence of confounding variables instead of a traditional overall survival analysis.

\section{Conclusion}

When conducting population-based cohort studies, a CR analysis is recommended for cancers with short survival and high mortality. The different age subgroups had their own independent determinants of tumor-induced mortality. The CR nomogram represents the first attempt at a predictive model for quantifying tumor-induced 
mortality in PS-GBM. However, due to the inherent limitations of the SEER dataset, our conclusions must be validated by multicenter prospective studies before they can be applied to clinical setting.

\section{Summary points}

- For population-based cohort studies, a competing risk (CR) analysis is recommended for cancers with short survival and high mortality.

- Machine learning methods such as conditional inference tree (ctree) and bootstrap were applied in the CR analysis.

- For glioblastoma multiforme (GBM), the prognostic effects of multilobe infiltration are only present in young adult patients.

- Social factors, such as marital status and socioeconomic status, significantly influence GBM-induced mortality in elderly patients between 60 and 78 years.

- The resection extent, radiotherapy and tumor size are independent determinants of GBM-induced mortality, regardless of the age subgroup.

- The benefit of the administration of chemotherapy is not significant in older patients in this cohort.

- The CR nomogram represents the first attempt at a predictive model for quantifying tumor-induced mortality in adult GBM patients.

\section{Supplementary data}

To view the supplementary data that accompany this paper please visit the journal website at:

https://www.futuremedicine.com/doi/suppl/10.2217/fon-2018-0719

\section{Acknowledgements}

Thanks are due to the efforts of the SEER Program tumor registries in providing high-quality open resources for researchers.

Financial \& competing interests disclosure

The study was supported by the Foundation of Tianjin Science and Technology Committee (14JCZDJC35600) and the National Key Technology Support Program (2014BAI04B00). The authors have no other relevant affiliations or financial involvement with any organization or entity with a financial interest in or financial conflict with the subject matter or materials discussed in the manuscript apart from those disclosed.

No writing assistance was utilized in the production of this manuscript.

\section{Ethical conduct of research}

This article does not contain any studies with human participants performed by any of the authors. No ethics approval was sought for this study by authors as data used were the National Cancer Institute's SEER database, which is a publicly available de-identified population-based database.

\section{Data sharing statement}

The authors certify that this manuscript reports the secondary analysis of clinical trial data that have been shared with them, and that the use of this shared data is in accordance with the terms (if any) agreed upon their receipt. The source of this data is: SEER.

\section{Open access}

This work is licensed under the Attribution-NonCommercial-NoDerivatives 4.0 Unported License. To view a copy of this license, visit http://creativecommons.org/licenses/by-nc-nd/4.0/

\section{References}

1 Ohgaki H, Kleihues P. The definition of primary and secondary glioblastoma. Clin. Cancer Res. 19(4), 764-772 (2013).

2 Alifieris C, Trafalis DT. Glioblastoma multiforme: pathogenesis and treatment. Pharmacol. Ther. 152, 63-82 (2015).

3 Chakrabarti I, Cockburn M, Cozen W, Wang YP, Preston-Martin S. A population-based description of glioblastoma multiforme in Los Angeles County, 1974-1999. Cancer 104(12), 2798-2806 (2005).

4 Wrensch M, Minn Y, Chew T, Bondy M, Berger MS. Epidemiology of primary brain tumors: current concepts and review of the literature. Neuro Oncol. 4(4), 278-299 (2002). 
5 Kim HT. Cumulative incidence in competing risks data and competing risks regression analysis. Clin. Cancer Res. 13(2 Pt 1), 559-565 (2007).

6 Camp RL, Dolled-Filhart M, Rimm DL. X-tile: a new bio-informatics tool for biomarker assessment and outcome-based cut-point optimization. Clin. Cancer Res. 10(21), 7252-7259 (2004).

7 Wachtel MS, Yang S. Odds of death after glioblastoma diagnosis in the United States by chemotherapeutic era. Cancer Med. 3(3), 660-666 (2014).

8 Zhu P, Du XL, Lu G, Zhu JJ. Survival benefit of glioblastoma patients after FDA approval of temozolomide concomitant with radiation and bevacizumab: a population-based study. Oncotarget 8(27), 44015-44031 (2017).

9 Yu M, Tatalovich Z, Gibson JT, Cronin KA. Using a composite index of socioeconomic status to investigate health disparities while protecting the confidentiality of cancer registry data. Cancer Causes Control. 25(1), 81-92 (2014).

10 Noorbakhsh A, Tang JA, Marcus LP et al. Gross-total resection outcomes in an elderly population with glioblastoma: a SEER-based analysis. J. Neurosurg. 120(1), 31-39 (2014).

11 Kattan MW, Heller G, Brennan MF. A competing-risks nomogram for sarcoma-specific death following local recurrence. Stat. Med. 22(22), 3515-3525 (2003).

12 Blanche P, Proust-Lima C, Loubere L, Berr C, Dartigues JF, Jacqmin-Gadda H. Quantifying and comparing dynamic predictive accuracy of joint models for longitudinal marker and time-to-event in presence of censoring and competing risks. Biometrics 71(1), $102-113(2015)$.

13 Noordzij M, Leffondre K, Van Stralen KJ, Zoccali C, Dekker FW, Jager KJ. When do we need competing risks methods for survival analysis in nephrology? Nephrol. Dial. Transplant. 28(11), 2670-2677 (2013).

14 Lau B, Cole SR, Gange SJ. Competing risk regression models for epidemiologic data. Am. J. Epidemiol. 170(2), $244-256$ (2009).

15 Ho AS, Wang L, Palmer FL et al. Postoperative nomogram for predicting cancer-specific mortality in medullary thyroid cancer. Ann. Surg. Oncol. 22(8), 2700-2706 (2015).

16 Li D, Zhong C, Tang X, Yu L, Ding K, Yuan Y. Competing nomograms help in the selection of elderly patients with colon cancer for adjuvant chemotherapy. J. Cancer Res. Clin. Oncol. 144(5), 909-923 (2018).

17 Shen W, Sakamoto N, Yang L. Cancer-specific mortality and competing mortality in patients with head and neck squamous cell carcinoma: a competing risk analysis. Ann. Surg. Oncol. 22(1), 264-271 (2015).

18 Zhou H, Zhang Y, Qiu Z et al. Nomogram to predict cause-specific mortality in patients with surgically resected stage I non-small-cell lung cancer: a competing risk analysis. Clin. Lung Cancer 19(2), e195-e203 (2018).

19 Stupp R, Mason WP, Van Den Bent MJ et al. Radiotherapy plus concomitant and adjuvant temozolomide for glioblastoma. $N$. Engl. J. Med. 352(10), 987-996 (2005).

20 Brown TJ, Brennan MC, Li M et al. Association of the extent of resection with survival in glioblastoma: a systematic review and meta-analysis. JAMA Oncol. 2(11), 1460-1469 (2016).

21 Keime-Guibert F, Chinot O, Taillandier L et al. Radiotherapy for glioblastoma in the elderly. N. Engl. J. Med. 356(15), 1527-1535 (2007).

22 Parsa AT, Wachhorst S, Lamborn KR et al. Prognostic significance of intracranial dissemination of glioblastoma multiforme in adults. $J$. Neurosurg. 102(4), 622-628 (2005).

23 Chang SM, Barker FG 2nd. Marital status, treatment, and survival in patients with glioblastoma multiforme: a population based study. Cancer 104(9), 1975-1984 (2005).

24 Xie JC, Yang S, Liu XY, Zhao YX. Effect of marital status on survival in glioblastoma multiforme by demographics, education, economic factors, and insurance status. Cancer Med. 7(8), 3722-3742 (2018).

25 Deb S, Pendharkar AV, Schoen MK, Altekruse S, Ratliff J, Desai A. The effect of socioeconomic status on gross total resection, radiation therapy and overall survival in patients with gliomas. J. Neurooncol. 132(3), 447-453 (2017).

26 Porter AB, Lachance DH, Johnson DR. Socioeconomic status and glioblastoma risk: a population-based analysis. Cancer Causes Control 26(2), 179-185 (2015).

27 Iasonos A, Schrag D, Raj GV, Panageas KS. How to build and interpret a nomogram for cancer prognosis. J. Clin. Oncol. 26(8), 1364-1370 (2008). 